\title{
WIDE-AREA BASED ON COORDINATED TUNING OF FUZZY PSS AND FACTS CONTROLLER IN MULTI-MACHINE ENVIRONMENT
}

\author{
Homayoun Ebrahimian ${ }^{1}$, Ebrahim Farzaneh Sheikhahmad ${ }^{2, *}$ \\ 1 Department of Basic Sciences, School of Medicine, Ardabil University of Medical Sciences, Ardabil, Iran \\ 2 Department of Electrical Engineering, Ardabil Branch, Islamic Azad University, Ardabil, Iran \\ * Corresponding author e-mail: eng.farzaneh@gmail.com
}

Received: 2015.12.15

Accepted: 2016.02.01

Published: 2016.03.01

\begin{abstract}
In this paper coordination of fuzzy power system stabilizer (FPSS) and flexible ac transmission systems (FACTS) have been considered in a multi-machine power system. The proposed model, has been applied for a wide-area power system. The proposed FPSS presented with local, nonlinear feedbacks, and the corresponding control synthesis conditions are given in terms of solutions to a set of linear matrix inequalities (LMIs). For this model, in fuzzy control synthesis, the new proposed control design method is based on fewer fuzzy rules and less computational burden. Also, the parameters of FACTS controller have been evaluated by improved honey bee mating optimization (IHBMO). The effectiveness of the proposed method has been applied over two case studies of single-machine infinite-bus (SMIB) and two areas four machine (TAFM) Kundur's power system. The obtained results demonstrate the superiority of proposed strategy.
\end{abstract}

Keywords: wide area power system, FPSS, FACTS, improved honey bee mating optimization.

\section{INTRODUCTION}

By increasing the complexity of power grid interconnections, power systems may become increasingly vulnerable to low frequency oscillations, especially inter-area oscillations. Actually, damping effectiveness of local measurement based controls is limited because local measurements have limited modal observability [1-3]. In such situations, the use of wide-area signals in which the desired oscillatory modes may be readily observable could be more beneficial in damping inter-area oscillations of a large interconnected system. The ability and a potential to use wide-area signals for control purposes has increased since a significant investment has been made in the U.S. in deploying synchrophasor measurement technology.

These oscillations have also resulted in instability and blackouts in the power system. A traditional approach to damp out these oscillations is through conventional power system stabilizer (CPSS), forming part of the generator excitation system. Besides PSS, FACTS devices are also applied to enhance system stability. Particularly, in multi-machine systems, using only conventional PSS may not provide sufficient damping for inter-area oscillations. In these cases, FACTS controllers are effective solutions. These controllers usually employ local signals as inputs and may not always be effective to damp out the interarea modes of oscillations. But CPSSs cannot satisfy the power system stability enhancement. For this purpose intelligent methods have been applied for tuning the parameters of PSSs [4-7].

Recently, FPSS has been proposed for stability problem in multi-machine power systems. In this paper, Takagi-Sugeno (T-S) based fuzzy controller has been proposed as an FPSS controller. Actually, there are two types of methods to overcome the difficulty of classic T-S controller. One method is to exploit a good tradeoff be- 
tween the conservatism and the computational burden by reducing unimportant decision variables [8]. However, the obtained controllers are still with a number of control rules, which may be unfavorable for implementation. Another method is that the original nonlinear model is first simplified as much as possible. Then, a fuzzy model with fewer fuzzy rules is constructed based on the simplified nonlinear model by using a fuzzy local approximation technique [9]. However, the designed control laws based on the fuzzy model may not guarantee the stability of the original nonlinear system. In this paper, a class of T-S fuzzy models with local nonlinear models is exploited to describe the considered nonlinear systems. A new fuzzy control scheme with local nonlinear feedbacks is proposed, and the corresponding control synthesis conditions are developed in terms of solutions to a set of LMIs. In contrast to the existing methods for fuzzy control synthesis, the new proposed control design method is based on fewer fuzzy rules and less computational burden. Moreover, the local nonlinear feedback laws in the new fuzzy controllers are also helpful for achieving good control effects [10-12].

By considering the coordination of FPSS and FACTS we will evaluate the effects of these devices in power system stability by considering the wide-area coordinating (WAC). Accordingly, to enhance stability margins and control oscillatory modes by adding supplementary damping devices we will use the global remote signals which have been suggested since the introduction of the phasor measurement unit (PMU) technology. Remote signals transmit knowledge related to the overall network dynamics, in contrast with local signals, which often lack good observability of some significant inter-area modes [13]. Even though WAC controllers involve additional communication equipment, their implementation may turn out to be more cost effective than installing new control devices if the additional operating flexibility achieved in critical power systems compensates for the equipment cost [14-15].

\section{PROBLEM STATEMENT}

\section{Power system modeling}

In this paper a single machine and multimachine power systems are considered as a test cases where the third order model is presented in
[16-17]. Actually, the proposed power system consists of four generators and the electrical and mechanical part of $i^{\text {th }}$ generator is modeled as follow:

$$
\begin{aligned}
& \dot{\delta}_{i}(t)=w_{i}(t)-w_{0} \\
& \dot{w}_{i}(t)=\frac{w_{0}}{M_{i}}\left(P_{m i}-P_{e i}(t)\right)-\frac{D_{i}}{M_{i}}\left(w_{i}(t)-w_{0}\right) \\
& \dot{E}_{q i}(t)=\frac{1}{T_{d o i}^{\prime}}\left(E_{f i}(t)-E_{q i}(t)\right) \\
& E_{q i}(t)=E_{q i}^{\prime}(t)+\left(x_{d i}-x_{d i}^{\prime}\right) \cdot I_{d i}(t) \\
& V_{t i}(t)=\frac{1}{x_{d s i}}\left[x_{s i}^{2} E_{q i}^{2}+V_{s i}^{2} x_{q i}^{2}+2 x_{s i} x_{d i} x_{d s i} P_{e i} \cot \delta_{i}\right]^{1 / 2} \\
& P_{e i}(t)=\sum_{j=1}^{n} E_{q i}^{\prime}(t) E_{q j}^{\prime}(t)\left(B_{i j} \sin \left(\delta_{i j}(t)\right)+G_{i j} \cos \left(\delta_{i j}(t)\right)\right) \\
& Q_{e i}(t)=\sum_{j=1}^{n} E_{q i}^{\prime}(t) E_{q j}^{\prime}(t)\left(G_{i j} \sin \left(\delta_{i j}(t)\right)+B_{i j} \cos \left(\delta_{i j}(t)\right)\right) \\
& I_{d i}(t)=\sum_{j=1}^{n} E_{q j}^{\prime}(t)\left(G_{i j} \sin \left(\delta_{i j}(t)\right)-B_{i j} \cos \left(\delta_{i j}(t)\right)\right)=\frac{Q_{e i}(t)}{E_{q i}^{\prime}(t)} \\
& I_{q i}(t)=\sum_{j=1}^{n} E_{q j}^{\prime}(t)\left(B_{i j} \sin \left(\delta_{i j}(t)\right)-G_{i j} \cos \left(\delta_{i j}(t)\right)\right)=\frac{P_{e i}(t)}{E_{q i}^{\prime}(t)} \\
& E_{q i}(t)=x_{a d i} I_{f i}(t)
\end{aligned}
$$

Also, it can be presented after mathematical transformers where, $\Delta P_{e}, \Delta w$ and $\Delta V_{t}$ are quantities;

$\left[\begin{array}{c}\Delta \dot{P}_{e i} \\ \Delta \dot{w}_{i} \\ \Delta \dot{V}_{t i i}\end{array}\right]=\left[\begin{array}{ccc}\frac{S_{E i}-S_{V i}}{T_{d i}^{\prime} S_{V i}} & S_{E i}^{\prime} & -\frac{R_{V i} S_{E i}}{T_{d i} S_{V i}} \\ -\frac{w_{0 i}}{H_{i}} & -\frac{D_{i}}{H_{i}} & 0 \\ \frac{S_{E i}-S_{V i}}{T_{d i} R_{V i} S_{V i}} & \frac{S_{E i}^{\prime}-S_{V i}}{R_{V i}} & -\frac{S_{E i}}{T_{d i}^{\prime} S_{V i}}\end{array}\right]\left[\begin{array}{l}\Delta P_{e i} \\ \Delta w_{i} \\ \Delta V_{t i}\end{array}\right]+\left[\begin{array}{l}\frac{R_{E i}^{\prime}}{T_{d 0 i}} \\ 0 \\ \frac{R_{E i}^{\prime}}{T_{d 0 i} R_{V i}}\end{array}\right] \Delta E_{f d i}$

where: $\Delta P_{e i}-$ is the state deviation in generator electromagnetic power for the $i^{\text {th }}$ subsystem,

$\Delta \omega_{\mathrm{i}}-$ is the state deviation in rotor angular velocity for the ith subsystem, $\Delta V_{t i}$ - is the state deviation in the terminal voltage of the generator for the $i^{\text {th }}$ subsystem.

$$
\begin{gathered}
S_{E i}=\frac{E_{q i} U_{s i}}{X_{d \Sigma i}} \cos \delta_{i}+U_{s i}^{2} \frac{X_{d \Sigma i}-X_{q \Sigma i}}{X_{d \Sigma i} X_{q \Sigma i}} \cos 2 \delta_{i} \\
S_{E i}^{\prime}=\frac{E_{q i}^{\prime} U_{s i}}{X_{d \Sigma i}^{\prime}} \cos \delta_{i}+U_{s i}^{2} \frac{X_{d \Sigma i}^{\prime}-X_{q \Sigma i}}{X_{d \Sigma i}^{\prime} X_{q \Sigma i}} \cos 2 \delta_{i} \\
R_{E i}=\frac{U_{s i}}{X_{d \Sigma i}} \sin \delta_{i} \\
R_{E i}^{\prime}=\frac{U_{s i}}{X_{d \Sigma i}^{\prime}} \sin \delta_{i} \\
S_{V i}=S_{E i}-R_{V i} \frac{\partial U_{t i}}{\partial \delta_{i}}, R_{V i}=S_{E i} / \frac{\partial V_{t i}}{\partial E_{q i}}
\end{gathered}
$$


The linearized rotor motion equation for synchronous generator can be described as:

$$
T_{J} \frac{d \Delta w}{d t}=\Delta T_{m}-\Delta T_{e}-\Delta T_{D}
$$

where: $\Delta T_{m}-$ is the mechanical input torque,

$\Delta T_{e}$ - is the electromagnetic torque, and $\Delta T_{e}^{e}=K_{1} \Delta \delta+K_{2} \Delta \delta$. By neglecting the $K_{2} \Delta E_{q}^{\prime}$ the formulation can be described as; $\Delta T_{e}^{q}=K_{1} \Delta \delta+\Delta T_{D}$.

$D-$ is the natural damping constant.

Accordingly the above equation after Laplace transformer and $\Delta w=\mathrm{s} \Delta \delta / w_{0}$ can be described as:

$$
T_{J} \frac{s^{2} \Delta \delta}{w_{0}}=-K_{1} \Delta \delta-D \frac{s \Delta \delta}{w_{0}}
$$

Which can be described as:

$$
T_{J} s^{2}+D s+w_{0} K_{1}=0
$$

Or

$$
s^{2}+2 \zeta_{n} w_{n} s+w_{n}^{2}=0
$$

Accordingly, we can achieve the following equation from above equations:

$$
\begin{aligned}
& \zeta_{n}=D / 2 \sqrt{w_{0} T_{J} K_{1}} \\
& w_{n}=\sqrt{w_{0} K_{1} / T_{J}}
\end{aligned}
$$

where: $\xi_{n}-$ is the damping factor,

$w_{n}-$ is the un-damped mechanical oscillation frequency.

\section{STRUCTURE OF PROPOSED CONTROLLER}

\section{Fuzzy power system stabilizer}

Regarding some complexity in wide area power system and variation of the loads and network conditions, it can be considered as a multi-input and multi-output system. The T-S fuzzy controller is the best choice of this application. So, we can obtain the following T-S fuzzy model as:

$$
\begin{aligned}
& \text { IF } v_{1}(t) \text { is } \Gamma_{i 1} \text { and } v_{2}(t) \text { is } \Gamma_{i 2}, \ldots, v_{p}(t) \text { is } \Gamma_{i p} \\
& \text { THEN } x(t)=A_{i} x(t)+B_{1 i} \omega(t)+B_{2 i} u(t)+G_{i} \phi(t) \\
& z(t)=C_{1 i} x(t)+D_{1 i} \omega(t)+D_{2 i} u(t)+G_{z i} \phi(t)
\end{aligned}
$$

where: $i=1, \ldots, r . r$ is the number of IF-THEN rules, $v(t)=\left[v_{1}(t) v_{2}(t) \cdots v_{p}(t)\right] T \in R^{p \times 1}$ are the premise variables, and $\Gamma_{i j}$ are the fuzzy sets [18].

By using the fuzzy inference method with a singleton fuzzifier and product inference and cen- ter average defuzzifiers, the final T-S fuzzy model is obtained as follows:

$$
\begin{aligned}
& x(t)=\frac{\sum_{i=1}^{r} W_{i}(v(t))\left(A_{i} x(t)+B_{1 i} \omega(t)+B_{2 i} u(t)+G_{i} \phi(t)\right)}{\sum_{i=1}^{r} W_{i}(v(t))} \\
& z(t)=\frac{\sum_{i=1}^{r} W_{i}(v(t))\left(C_{1 i} x(t)+D_{1 i} \omega(t)+D_{2 i} u(t)+G_{z i} \phi(t)\right)}{\sum_{i=1}^{r} W_{i}(v(t))}
\end{aligned}
$$

Where $W_{i}(v(t))=\prod_{j=1}^{p} \eta_{i j}\left(v_{j}(t)\right) \cdot \eta_{i j}\left(v_{j}(t)\right)$ is the grade of membership of $v_{j}(t)$ in $\Gamma_{i j}$, where it is assumed that $\sum_{i=1}^{r} W_{i}(v(t))>0, W_{i}(v(t)) \geq 0, i=1,2, \ldots, r$. Denote $\alpha_{i}(v(t))=\left(\mathrm{w}_{i}(v(t))\right) /\left(\sum_{i=1}^{r} W_{i}(v(t))\right)$; then $0 \leq \alpha_{i}(v(t)) \leq 1$ and $\sum_{i=1}^{r} \alpha_{i}(v(t))=1$

Where $\alpha_{i}(v(t))$ is said to be normalized membership functions. Let $\alpha(v(t))=[\alpha 1(v(t))$, $\alpha 2(v(t))$, $\ldots, \alpha r(v(t))]^{T}$, and denote $\alpha(v(t))$ as $\alpha$ for a brief description. So, we can write:

$$
\begin{gathered}
\dot{x}(t)=A(\alpha) x(t)+B_{1}(\alpha) \omega(t)+B_{2}(\alpha) u(t)+G(\alpha) \phi(t) \\
z(t)=C_{1}(\alpha) x(t)+D_{1}(\alpha) \omega(t)+D_{2}(\alpha) u(t)+G_{z}(\alpha) \phi(t)
\end{gathered}
$$

with

$$
\begin{array}{ll}
A(\alpha)=\sum_{i=1}^{r} \alpha_{i}(t) A_{i} & B_{1}(\alpha)=\sum_{i=1}^{r} \alpha_{i}(t) B_{1 i} \\
B_{2}(\alpha)=\sum_{i=1}^{r} \alpha_{i}(t) B_{2 i} & G(\alpha)=\sum_{i=1}^{r} \alpha_{i}(t) G_{i} \\
C_{1}(\alpha)=\sum_{i=1}^{r} \alpha_{i}(t) C_{1 i} & D_{1}(\alpha)=\sum_{i=1}^{r} \alpha_{i}(t) D_{1 i} \\
D_{2}(\alpha)=\sum_{i=1}^{r} \alpha_{i}(t) D_{2 i} & G_{z}(\alpha)=\sum_{i=1}^{r} \alpha_{i}(t) G_{z i}
\end{array}
$$

In fact, $A(\alpha) x(t), B_{1}(\alpha), B_{2}(\alpha), G(\alpha), C_{1}(\alpha)$ $x(t), D_{1}(\alpha), D_{2}(\alpha)$, and $G_{z}(\alpha)$ in fuzzy model are the new descriptions of $\bar{f} a(x(t)), h(x(t)), g(x(t))$, $f_{b}(x(t)), \bar{f}_{z a}(x(t)), h_{z}(x(t)), g_{z}(x(t))$, and $f_{z b}(x(t))$ by fuzzy membership functions, respectively. The following lemma will be useful in the sequel.

Lemma 2: If the following conditions are hold:

$$
\begin{aligned}
M_{i i}<0, & 1 & \leq i \leq r \\
\frac{1}{r-1} M_{i i}+\frac{1}{2}\left(M_{i j}+M_{j i}\right)<0, & 1 & \leq i \neq j \leq r
\end{aligned}
$$

Then we can write;

$$
\begin{aligned}
& M_{i i}<0, \quad 1 \leq i \leq r \\
& \sum_{i=1}^{r} \sum_{j=1}^{r} \alpha_{i} \alpha_{j} M_{i i}<0 \\
& \text { where, } \alpha_{i}, 1 \leq i \leq r, \text { satisfy } 0 \leq \alpha_{i} \leq 1, \quad \sum_{=1}^{r} \alpha_{i}=1
\end{aligned}
$$




\section{Fuzzy PSS control strategy}

In this paper, the $H \infty$ control strategy and guaranteed cost control have been considered. Also, for local nonlinear feedbacks control and the corresponding control synthesis conditions has been solved by linear matrix inequalities (LMIs). We leave this section for preventing more description where, [19] presents further discussion in this field.

\section{Flexible AC transmission systems controller}

The structure of the proposed FACTS controller has been presented in Figure 1. Also, local signals of FACTS devices are applied for the damping control. In this paper, the active power flow through the series FACTS device line $P$ is employed [20-21].

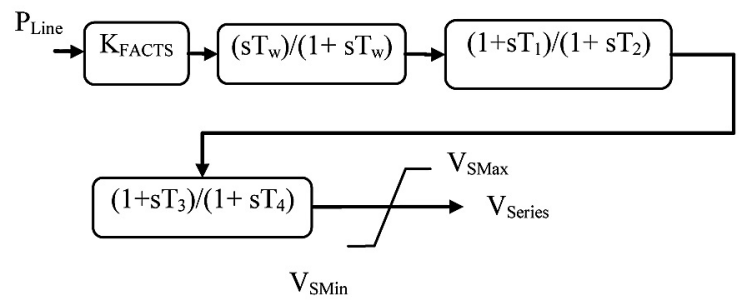

Fig. 1. The flowchart of FACTS controller

The parameters of the proposed FACTS controller have been optimized by IHBMO in this paper. For this purpose we introduce this algorithm in the next section

\section{IMPROVED HONEY BEE MATING OPTIMIZATION}

\section{Standard HBMO}

This section reviews briefly the original HBMO. In order to deepen the concept see ref [22]. At the start of the flight, the queen is initialized with some energy content and returns to her nest when her energy is within some threshold from zero or when her spermatheca is full. In developing the algorithm, the functionality of workers is restricted to brood care, and therefore, each worker may be represented as a heuristic which acts to improve and/or take care of a set of broods. A drone mates with a queen probabilistically using an annealing function as:

$$
\operatorname{prob}(Q, D)=e^{\frac{-\Delta(f)}{S(t)}}
$$

Where $\operatorname{Prob}(Q, D)$ is the probability of adding the sperm of drone $D$ to the spermatheca of queen $Q$ (that is, the probability of a successful mating); $\Delta(f)$ is the absolute difference between the fitness of $D$ (i.e., $f(D))$ and the fitness of $Q$ (i.e., $f(Q))$; and $S(t)$ is the speed of the queen at time $t$. It is apparent that this function acts as an annealing function, where the probability of mating is high when both the queen is still in the start of her mating-flight and therefore her speed is high, or when the fitness of the drone is as good as the queen's. After each transition in space, the queen's speed, $S(t)$, and energy, $E(t)$, decay using the following equations:

$$
\begin{aligned}
\mathrm{S}(\mathrm{t}+1) & =\alpha_{\text {нвмO }} \times \mathrm{S}(\mathrm{t}) \\
E(\mathrm{t}+1) & =E(\mathrm{t})-\gamma_{\text {HвMO }}
\end{aligned}
$$

where: $\alpha_{H B M O}(t)$ - is speed reduction factor and $\gamma_{\text {HBMO }}$ - is the amount of energy reduction after each transition $(\alpha, \gamma \in[0,1])$.

Thus, HBMO algorithm may be constructed with the following five main stages:

- Step 1: The algorithm starts with the mating flight, where a queen (best solution) selects drones probabilistically to form the spermatheca (list of drones). A drone is then selected from the list at random for the creation of broods.

- Step 2: Creation of new broods by crossoverring the drones' genotypes with the queen's (Breeding process). The breeding process can transfer the genes of drones and the queen to the $j^{\text {th }}$ individual [23].

$$
\text { child }=\text { parent }_{i}+\beta_{\text {HBMO }}\left(\text { parent }_{k}-\text { parent }_{i}\right)
$$

Where $\beta_{\text {HвMO }}$ is the decreasing factor $\left(\beta_{\text {HвMO }}\right.$ $\sim U(0,1))$.

- Step 3: Use of workers (heuristics) to conduct local search on broods (trial solutions).

- Step 4: Adaptation of workers' fitness based on the amount of improvement achieved on broods as follows:

$$
\begin{aligned}
& \text { Brood }_{i}^{k}=\text { Brood }_{i}^{k} \pm\left(\delta_{H B M O}+\varepsilon_{H B M O}\right) \text { Brood }_{i}^{k} \\
& \delta_{H B M O} \in[0,1], 0<\varepsilon_{H B M O}<1
\end{aligned}
$$

- Step 5: Replacement of weaker queens by fitter broods.

\section{Interactive honey bee mating optimization (IHBMO)}

A multi-objective optimization problem always has a set of optimal solutions, for which there is no way to improve one objective value 
without deterioration of at least one of the other objective values. Pareto dominance concept classifies solutions as dominated or non-dominated solutions and the "best solutions" are selected from the non-dominated solutions. To sort nondominated solutions, the first front of the nondominated solution is assigned the highest rank and the last one is assigned the lowest rank. When comparing solutions that belong to the same front, another parameter called crowding distance is calculated for each solution. The crowding distance is a measure of how close an individual is to its neighbors. Large average crowding distance will result in better diversity in the population [24].

\section{Fuzzy mechanism}

Upon having the Pareto-optimal set of nondominated solutions, the proposed approach presents one solution to the decision maker as the best compromise solutions. Due to imprecise nature of the decision maker's judgment, the $i^{\text {th }}$ objective function is represented by a membership function $\mu$ i defined as [25]:

$$
\mu_{i}\left(p_{g i}\right)=\frac{f_{i}^{\max }-f_{i}\left(p_{g i}\right)}{f_{i}^{\max }-f_{i}^{\min }}
$$

where: $f_{i}^{\max }$ and $f_{i}^{\min }$ are the maximum and minimum values of $i^{\text {th }}$ objective, respectively.

$$
F D M_{i}\left(p_{g i}\right)=\left\{\begin{array}{cc}
0 & \mu_{i}\left(p_{g i}\right) \leq 0 \\
\mu_{i}\left(p_{g i}\right) & 0<\mu_{i}\left(p_{g i}\right)<1 \\
1 & \mu_{i}\left(p_{g i}\right) \geq 1
\end{array}\right.
$$

For each non-dominated solution $k$, the normalized membership function $F D M^{k}$

$$
F D M^{k}=\left[\frac{\sum_{i=1}^{2} F D M_{i}^{k}\left(p_{g i}\right)}{\sum_{j=1}^{M} \sum_{i=1}^{2} F D M_{i}^{j}}\right]
$$

The best compromise solution of stability problem is the one having the maximum value of $F D M^{k}$ as a fuzzy decision making function. Where $M$ is the total number of non-dominated solutions. Then all the solutions are arranged in descending order according to their membership function values which will guide the decision makers with a priority list of non-dominated solutions in view of the current operating conditions. Figure 2 shows the membership structure $\mu_{c}$ for the fuzzy logical variable signifying the total fuel $\operatorname{cost} f_{i}\left(P_{g i}\right)$. Also, the flowchart of the proposed method has been presented in Figure 3.

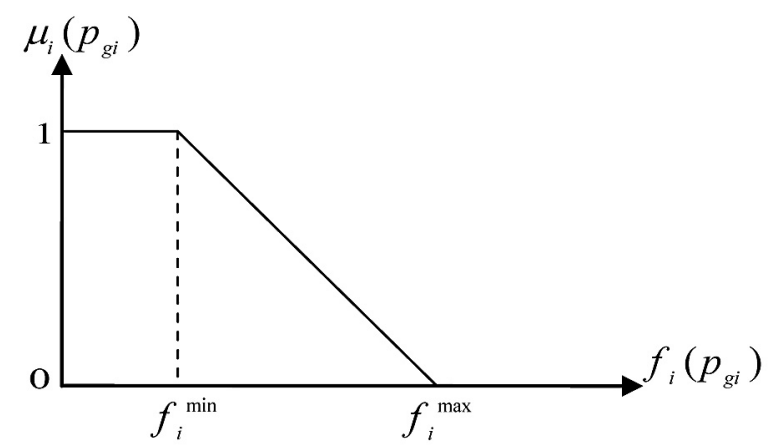

Fig. 2. Membership function of fuzzy fuel cost

\section{NUMERICAL RESULTS AND DISCUSSION}

\section{One machine infinite bus}

For stability assessment of power system adequate mathematical models describing the system are needed. The system behaviour following such a disturbance is critically dependent upon the magnitude, nature and the location of fault and to a certain extent on the system operating conditions. A schematic diagram for the proposed first test case is presented in Figure 4.

The disturbances are given at $t=1 \mathrm{sec}$. System responses in a form of slip $\left(S_{m}\right)$ are plotted. The following types of disturbances have been considered [26]:

- Scenario 1: A step change of $0.1 \mathrm{pu}$ in the input mechanical torque.

- Scenario 2: A three phase-to-ground fault for $100 \mathrm{~ms}$ at the generator terminal.

The convergence trend of proposed algorithm is presented in Figure 5. Also, the achieved results for FACTS controller are presented in Table 1.

In the following, Figure 6 presents the system response at the lagging power factor operating conditions with weak transmission system for scenario 1 . It is clear that the proposed method could provide better stability conditions. In this test case, the proposed model has been compared with other three algorithms which have been resented in [26]. The mentioned algorithms are particle swarm optimization (PSO) and two other versions of this algorithm, named PSO-TVAC and PSO-TVIW [27]. Also, Figure 7 presents the system response in scenario 2 with inertia $H^{\prime}=H / 4$.

Furthermore, to demonstrate the robustness performance of the proposed method, in the some operating condition for scenario 1, the Eigen values of the system with comparison of these methods has been presented in Table 2. All of the presented numerical results have been quoted direct- 


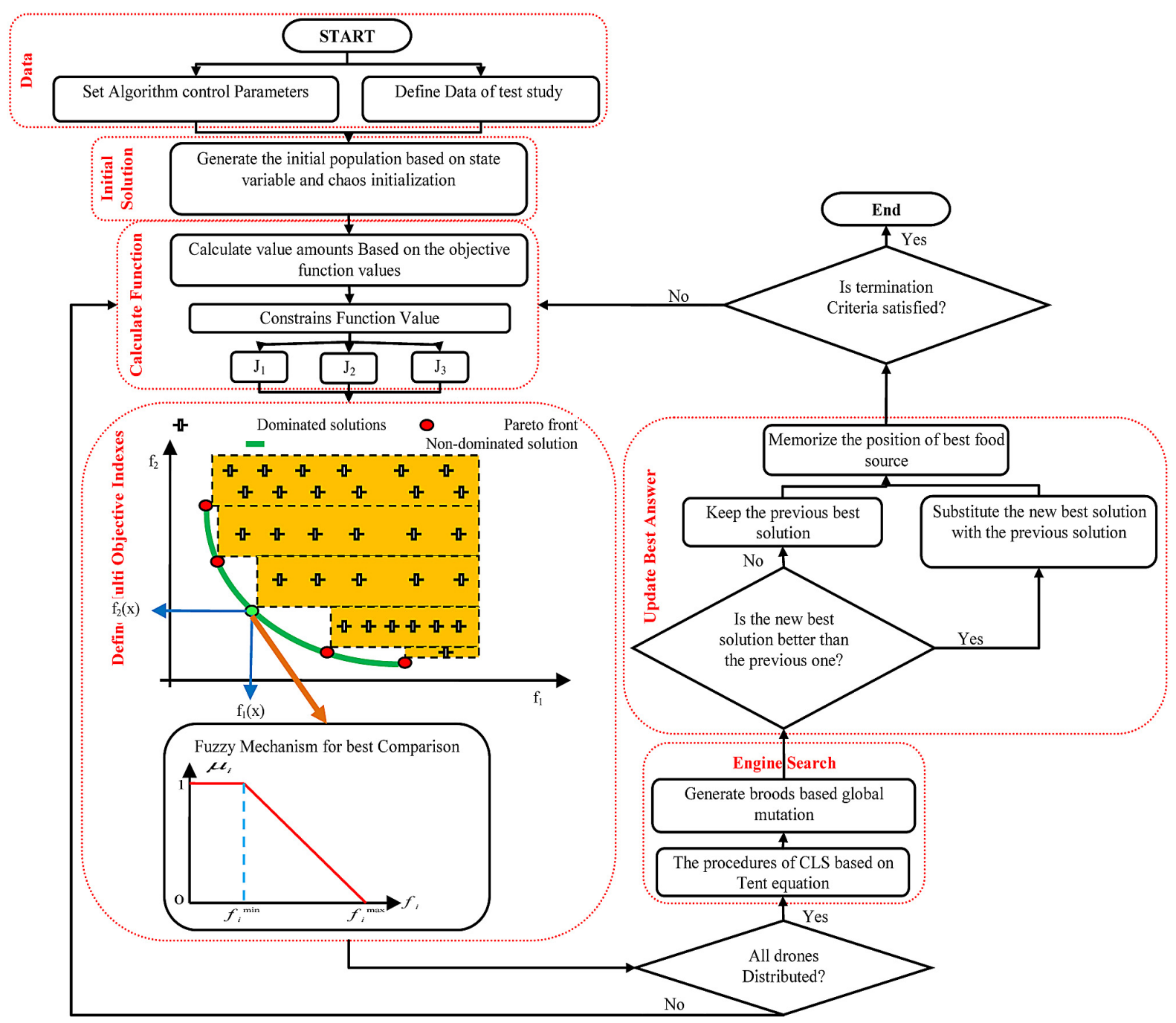

Fig. 3. Flowchart of proposed IHBMO

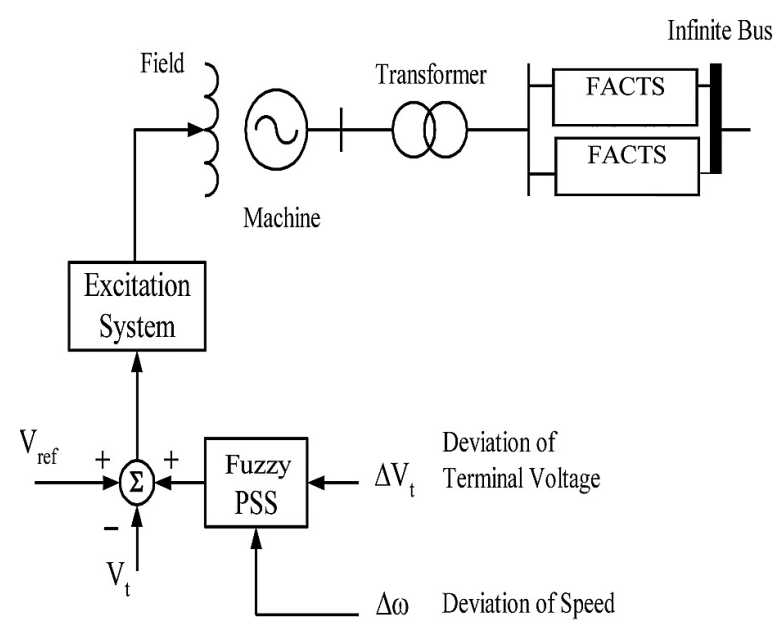

Fig. 4. Schematic diagram of one machine infinite bus system

Table 1. Parameters for proposed algorithms

\begin{tabular}{|c|c|c|c|c|}
\hline $\mathrm{K}$ & $\mathrm{T} 1$ & $\mathrm{~T} 2$ & $\mathrm{~T} 3$ & $\mathrm{~T} 4$ \\
\hline 31.66 & 0.0365 & 0.0274 & 0.0234 & 0.1467 \\
\hline
\end{tabular}

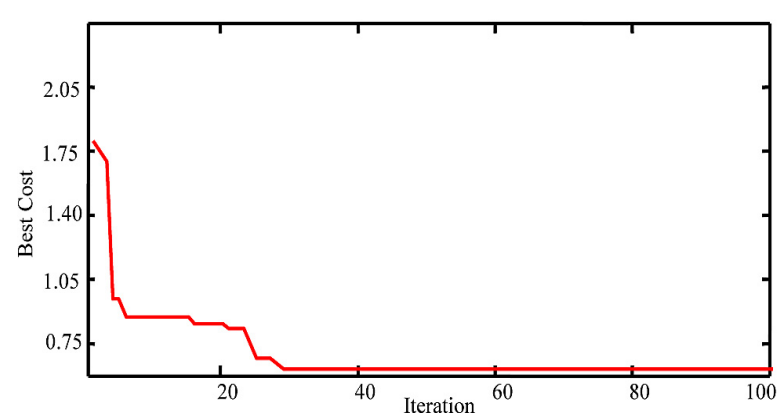

Fig. 5. Fitness convergence of proposed method

ly from [26]. It is clear to see that the eigenvalues of the system with the proposed model are farther than the imaginary axis and the system stability margin is more than other methods.

For more information about the proposed algorithm the computational results which are used in this paper through several runs of the proposed technique. The computational results are shown in Table 3. 
Table 2. The Eigen values of system with different PID for scenario 1

\begin{tabular}{|c|c|c|c|c|c|c|c|c|}
\hline $\begin{array}{c}\text { Case } \\
\text { No. }\end{array}$ & $\mathrm{P}$ & Q & $X_{e}$ & $\mathrm{H}$ & PSO & PSO-TVIW & PSO-TVAC & Proposed \\
\hline 1 & 0.8 & 0.4 & 0.3 & 3.25 & $\begin{array}{c}-1.0097 \\
-0.0070+0.1577 \mathrm{i} \\
-0.0070-0.1577 \mathrm{i} \\
-0.1573 \\
-0.0242 \\
-0.0005\end{array}$ & $\begin{array}{c}-1.0054 \\
-0.1287 \\
-0.0192+0.0715 \mathrm{i} \\
-0.0192-0.0715 \mathrm{i} \\
-0.0326 \\
-0.0008\end{array}$ & $\begin{array}{c}-1.0077 \\
-0.1065 \\
-0.0277+0.0728 \mathrm{i} \\
-0.0277-0.0728 \mathrm{i} \\
-0.0355 \\
-0.0010\end{array}$ & $\begin{array}{c}-1.0053 \\
-0.1232 \\
-0.0144+0.0586 \mathrm{i} \\
-0.0144-0.0586 \mathrm{i} \\
-0.0324 \\
-0.0007\end{array}$ \\
\hline 2 & 0.5 & 0.1 & 0.3 & 3.25 & $\begin{array}{c}-1.0088 \\
-0.0096+0.1480 \mathrm{i} \\
-0.0096-0.1480 \mathrm{i} \\
-0.1584 \\
-0.0188 \\
-0.0005\end{array}$ & $\begin{array}{c}-1.0049 \\
-0.1298 \\
-0.0228+0.0659 i \\
-0.0228-0.0659 i \\
-0.0249 \\
-0.0008\end{array}$ & $\begin{array}{c}-1.0069 \\
-0.1099 \\
-0.0312+0.0672 \mathrm{i} \\
-0.0312-0.0672 \mathrm{i} \\
-0.0260 \\
-0.0010\end{array}$ & $\begin{array}{c}-1.0036 \\
-0.1257 \\
-0.0200+0.0616 \mathrm{i} \\
-0.0200-0.0616 \mathrm{i} \\
-0.0240 \\
-0.0007\end{array}$ \\
\hline 3 & 1.0 & 0.5 & 0.3 & 3.25 & $\begin{array}{c}-1.0106 \\
-0.0054+0.1620 \mathrm{i} \\
-0.0054-0.1620 \mathrm{i} \\
-0.1565 \\
-0.0273 \\
-0.0005\end{array}$ & $\begin{array}{c}-1.0059 \\
-0.1277 \\
-0.0171+0.0739 \mathrm{i} \\
-0.0171-0.0739 \mathrm{i} \\
-0.0373 \\
-0.0008\end{array}$ & $\begin{array}{c}-1.0084 \\
-0.0260+0.0752 \mathrm{i} \\
-0.0260-0.0752 \mathrm{i} \\
-0.1026 \\
-0.0420 \\
-0.0010\end{array}$ & $\begin{array}{c}-1.0031 \\
-0.1183 \\
-0.0096+0.0602 \mathrm{i} \\
-0.0096-0.0602 \mathrm{i} \\
-0.0427 \\
-0.0007\end{array}$ \\
\hline 4 & 0.8 & 0.4 & 0.6 & 3.25 & $\begin{array}{c}-1.0077 \\
-0.0090+0.1309 i \\
-0.0090-0.1309 i \\
-0.1362 \\
-0.0422 \\
-0.0005\end{array}$ & $\begin{array}{c}-1.0042 \\
-0.0125+0.0524 i \\
-0.0125-0.0524 i \\
-0.0874+0.0267 i \\
-0.0874-0.0267 i \\
-0.0008\end{array}$ & $\begin{array}{c}-1.0061 \\
-0.0827+0.0465 \mathrm{i} \\
-0.0827-0.0465 \mathrm{i} \\
-0.0163+0.0492 \mathrm{i} \\
-0.0163-0.0492 \mathrm{i} \\
-0.0011\end{array}$ & $\begin{array}{c}-1.0040 \\
-0.0765+0.0276 i \\
-0.0765-0.0276 i \\
-0.0101+0.0487 i \\
-0.0101-0.0487 i \\
-0.0008\end{array}$ \\
\hline 5 & 0.5 & 0.1 & 0.6 & 3.25 & $\begin{array}{c}-1.0069 \\
-0.0120+0.1297 \mathrm{i} \\
-0.0120-0.1297 \mathrm{i} \\
-0.1388 \\
-0.0343 \\
-0.0005\end{array}$ & $\begin{array}{c}-1.0038 \\
-0.0168+0.0487 \mathrm{i} \\
-0.0168-0.0487 \mathrm{i} \\
-0.0833+0.0219 \mathrm{i} \\
-0.0833-0.0219 \mathrm{i} \\
-0.0008\end{array}$ & $\begin{array}{c}-1.0054 \\
-0.0791+0.0439 \mathrm{i} \\
-0.0791-0.0439 \mathrm{i} \\
-0.0202+0.0447 \mathrm{i} \\
-0.0202-0.0447 \mathrm{i} \\
-0.0011\end{array}$ & $\begin{array}{c}-1.0020 \\
-0.0793+0.0256 \mathrm{i} \\
-0.0793-0.0256 \mathrm{i} \\
-0.0143+0.0472 \mathrm{i} \\
-0.0143-0.0472 \mathrm{i} \\
-0.0007\end{array}$ \\
\hline 6 & 1.0 & 0.5 & 0.6 & 3.25 & $\begin{array}{c}-1.0084 \\
-0.0067+0.1293 \mathrm{i} \\
-0.0067-0.1293 \mathrm{i} \\
-0.1339 \\
-0.0484 \\
-0.0005\end{array}$ & $\begin{array}{c}-1.0046 \\
-0.0904+0.0292 \mathrm{i} \\
-0.0904-0.0292 \mathrm{i} \\
-0.0093+0.0535 \mathrm{i} \\
-0.0093-0.0535 \mathrm{i} \\
-0.0008\end{array}$ & $\begin{array}{c}-1.0066 \\
-0.0856+0.0485 i \\
-0.0856-0.0485 i \\
-0.0131+0.0507 i \\
-0.0131-0.0507 i \\
-0.0011\end{array}$ & $\begin{array}{c}-1.0044 \\
-0.0876+0.0318 \mathrm{i} \\
-0.0876-0.0318 \mathrm{i} \\
-0.0052+0.0512 \mathrm{i} \\
-0.0052-0.0512 \mathrm{i} \\
-0.0008\end{array}$ \\
\hline 7 & 0.8 & 0.0 & 0.6 & 3.25 & $\begin{array}{c}-1.0098 \\
-0.0051+0.1443 \mathrm{i} \\
-0.0051-0.1443 \mathrm{i} \\
-0.1445 \\
-0.0395 \\
-0.0005\end{array}$ & $\begin{array}{c}-1.0054 \\
-0.0121+0.0621 \mathrm{i} \\
-0.0121-0.0621 \mathrm{i} \\
-0.1052 \\
-0.0690 \\
-0.0008\end{array}$ & $\begin{array}{c}-1.0077 \\
-0.0791+0.0342 \mathrm{i} \\
-0.0791-0.0342 \mathrm{i} \\
-0.0191+0.0596 \mathrm{i} \\
-0.0191-0.0596 \mathrm{i} \\
-0.0011\end{array}$ & $\begin{array}{c}-1.0051 \\
-0.0103+0.0599 i \\
-0.0103-0.0599 i \\
-0.0975 \\
-0.0807 \\
-0.0006\end{array}$ \\
\hline 8 & 1.0 & -0.2 & 0.3 & 3.25 & $\begin{array}{c}-1.0147 \\
0.0029+0.1753 \mathrm{i} \\
0.0029-0.1753 \mathrm{i} \\
-0.1534 \\
-0.0427 \\
-0.0005\end{array}$ & $\begin{array}{c}-1.0082 \\
-0.1295 \\
-0.0057+0.0825 \mathrm{i} \\
-0.0057-0.0825 \mathrm{i} \\
-0.0560 \\
-0.0008\end{array}$ & $\begin{array}{c}-1.0117 \\
-0.0152+0.0838 \mathrm{i} \\
-0.0152-0.0838 \mathrm{i} \\
-0.0815+0.0127 \mathrm{i} \\
-0.0815-0.0127 \mathrm{i} \\
-0.0011\end{array}$ & $\begin{array}{c}-1.0063 \\
-0.1254 \\
-0.0038+0.0699 \mathrm{i} \\
-0.0038+0.0699 \mathrm{i} \\
-0.0601 \\
-0.0007\end{array}$ \\
\hline 9 & 0.5 & -0.2 & 0.6 & 3.25 & $\begin{array}{c}-1.0092 \\
-0.0094+0.1432 \mathrm{i} \\
-0.0094-0.1432 \mathrm{i} \\
-0.1489 \\
-0.0271 \\
-0.0005\end{array}$ & $\begin{array}{c}-1.0051 \\
-0.1108 \\
-0.0209+0.0592 \mathrm{i} \\
-0.0209-0.0592 \mathrm{i} \\
-0.0463 \\
-0.0008\end{array}$ & $\begin{array}{c}-1.0072 \\
-0.0311+0.0561 \mathrm{i} \\
-0.0311-0.0561 \mathrm{i} \\
-0.0673+0.0191 \mathrm{i} \\
-0.0673-0.0191 \mathrm{i} \\
-0.0010\end{array}$ & $\begin{array}{c}-1.0043 \\
-0.1054 \\
-0.0176+0.0533 i \\
-0.0176+0.0533 i \\
-0.0536 \\
-0.0007\end{array}$ \\
\hline 10 & 1.0 & 0.2 & 0.3 & 0.81 & $\begin{array}{c}-1.0440 \\
0.0103+0.3371 \mathrm{i} \\
0.0103-0.3371 \mathrm{i} \\
-0.1531 \\
-0.0287 \\
-0.0005\end{array}$ & $\begin{array}{c}-1.0260 \\
-0.0256+0.1867 \mathrm{i} \\
-0.0256-0.1867 \mathrm{i} \\
-0.0890 \\
-0.0390 \\
-0.0008\end{array}$ & $\begin{array}{c}-1.0363 \\
-0.0328+0.2094 i \\
-0.0328-0.2094 i \\
-0.0516+0.0069 i \\
-0.0516-0.0069 i \\
-0.0010\end{array}$ & $\begin{array}{c}-1.0223 \\
-0.0252+0.180 \mathrm{i} \\
-0.0252+0.180 \mathrm{i} \\
-0.0806 \\
-0.0427 \\
-0.0007\end{array}$ \\
\hline
\end{tabular}



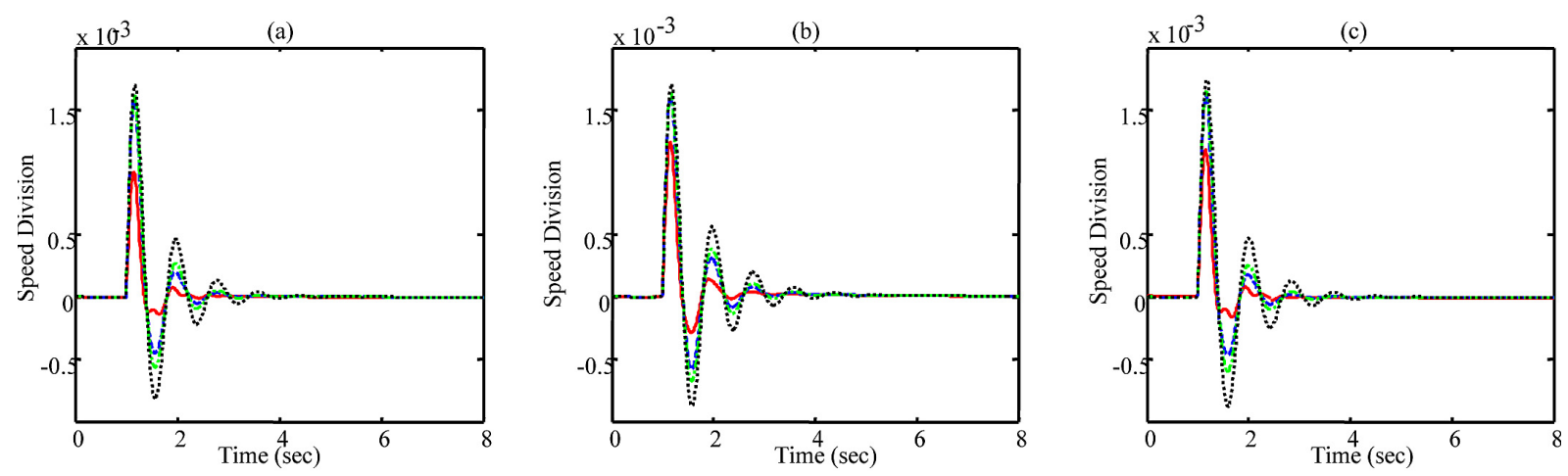

Fig. 6. $\Delta \mathrm{T}_{\mathrm{m}}=0.1$ (p.u.) under $\mathrm{X}_{\mathrm{e}}=0.3$; PSO (dotted), PSO-TVIW (dashed-doted), PSO-TVAC (dashed) and proposed (solid) a) $\mathrm{P}=0.8, \mathrm{Q}=0.4$ b) $\mathrm{P}=0.5, \mathrm{Q}=0.1$ c) $\mathrm{P}=1.0, \mathrm{Q}=0.5$
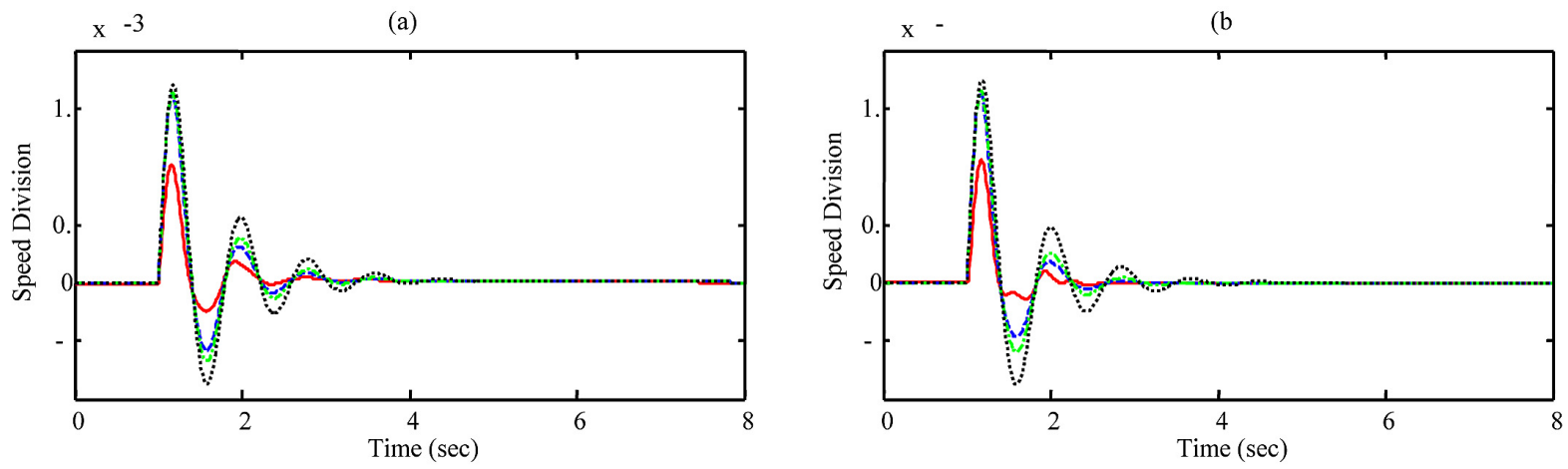

Fig. 7. $\Delta \mathrm{T}_{\mathrm{m}}=0.1$ (p.u.) under $\mathrm{Xe}=0.6$ and $\mathrm{H}^{\prime}=\mathrm{H} / 4$; PSO (dotted), PSO-TVIW (dashed-doted), PSO-TVAC (dashed) and proposed (solid) a) $\mathrm{P}=1.0, \mathrm{Q}=0.5 \mathrm{~b}$ ) $\mathrm{P}=0.6, \mathrm{Q}=0.0$

Table 3. The average results over many runs of proposed algorithm

\begin{tabular}{|c|c|c|c|c|c|c|c|c|c|c|c|c|c|c|c|c|}
\hline \multirow{2}{*}{ Run } & \multicolumn{4}{|c|}{ PSO } & \multicolumn{4}{|c|}{ PSO-TVIW } & \multicolumn{4}{|c|}{ PSO-TVAC } & \multicolumn{4}{|c|}{ Proposed } \\
\hline & Min & Mean & Max & Iter & Min & Mean & Max & Iter & Min & Mean & Max & Iter & Min & Mean & Max & Iter \\
\hline 1 & 0.456 & 1.465 & 2.154 & 85 & 0.409 & 0.721 & 1.565 & 53 & 0.401 & 0.675 & 1.321 & 36 & 0.401 & 0.630 & 1.128 & 24 \\
\hline 2 & 0.412 & 1479 & 024 & 65 & 0 & 19 & 1.570 & 6 & 406 & 678 & 326 & 30 & 404 & .630 & 125 & 25 \\
\hline 3 & 0.466 & 1 & 01 & 80 & 0 & 0.722 & 1.569 & 57 & .402 & 0.669 & 1.330 & 41 & .401 & 31 & 1.1 & 27 \\
\hline 4 & 0.419 & 1.496 & 2.063 & 91 & 0.412 & 723 & 1.566 & 62 & 0.401 & 0.675 & 1.327 & 35 & 0.401 & 0.631 & 1.129 & 29 \\
\hline 5 & 0.455 & 1.488 & 2.130 & 76 & 0.401 & 0.731 & 1.568 & 63 & 0.403 & 0.671 & 1.326 & 29 & 0.402 & 0.630 & 1.125 & 29 \\
\hline 6 & 0.401 & 1.506 & 2.114 & 85 & 0.422 & 0.725 & 1.563 & 59 & 0.409 & 0.669 & 1.325 & 25 & 0.402 & 0.630 & 1.12 & 24 \\
\hline 7 & 0.462 & 1.502 & 2.213 & 78 & 0.431 & 0.719 & 1.561 & 64 & 0.401 & 0.673 & 1.332 & 32 & 0.402 & 0.636 & 1.125 & 30 \\
\hline 8 & 0.476 & 1.479 & 2.006 & 69 & 0.41 & 0.711 & 1.562 & $5 \mathrm{~s}$ & 0.410 & 0.665 & 1.331 & 3. & 0.402 & 0.631 & 1.125 & 24 \\
\hline 9 & 186 & 36 & 36 & 9 & 0.408 & 22 & 1 & 65 & 1 & 52 & 1.327 & 24 & 1 & 1 & $6-1$ & 7 \\
\hline 10 & 0.445 & 1.462 & 2.116 & 56 & 0.413 & 0.720 & 1.562 & 58 & 0.406 & 0.662 & 1.331 & 26 & 0.403 & 0.631 & 1.127 & 24 \\
\hline SD & 0.026 & 0.016 & 0.064 & 11.48 & 0.008 & 0.004 & 0.003 & 3.44 & 0.003 & 0.007 & 0.003 & 5.06 & 0.001 & 0.006 & 0.002 & 2.63 \\
\hline
\end{tabular}

\section{Two-area four-machine system}

Kundur's two-area four-machine (TAFM) system consisting of two fully symmetrical areas linked together by two $220 \mathrm{~km}, 230 \mathrm{kV}$ transmission lines [27] is considered as a first case study in this manuscript as shown in Figure 8. This power system typically is used to study the low frequency electromechanical oscillations of a large interconnected system. In this system, each area consists of two generators, connected with tie-lines between buses 7-8 and 8-9. Where, each generator is assumed to be connected with governors, Automatic voltage regulator (AVR) and IEEE ST1A type static exciter. The classic PSS is connected to $G_{2}$ and $G_{3}$ based on and intelligent method presented in [28] to damp out local modes of oscillations. Then, the additional global signal is assumed to be provided to the stabilizer of the selected generator from a centralized wide 


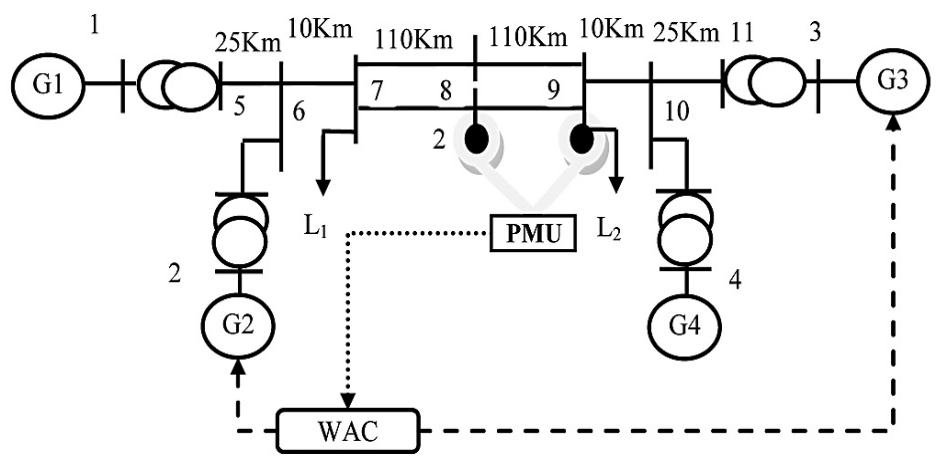

Fig. 8. TAFM power system

a)

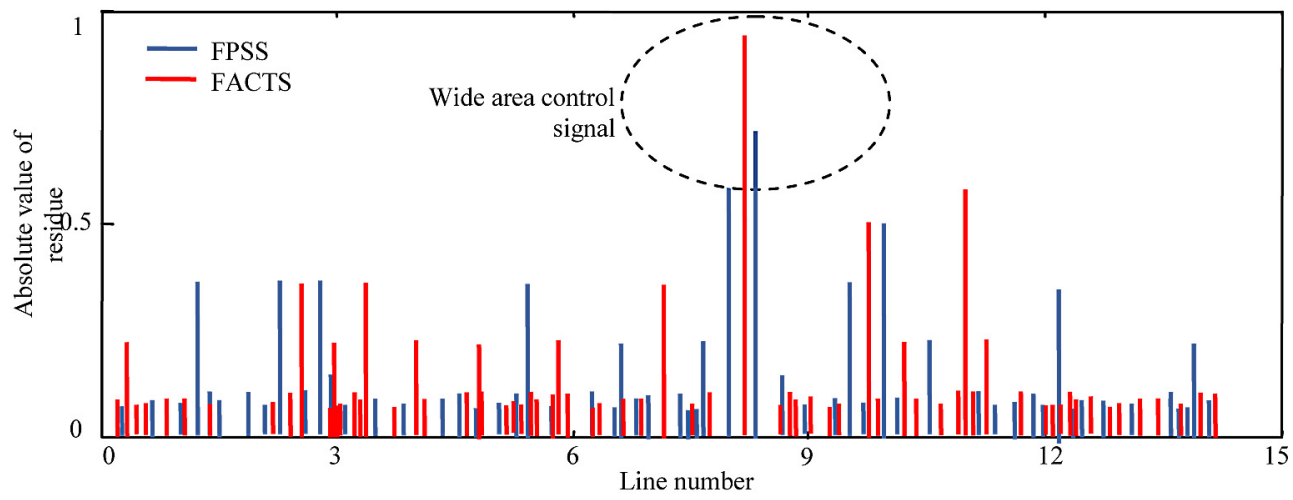

b)

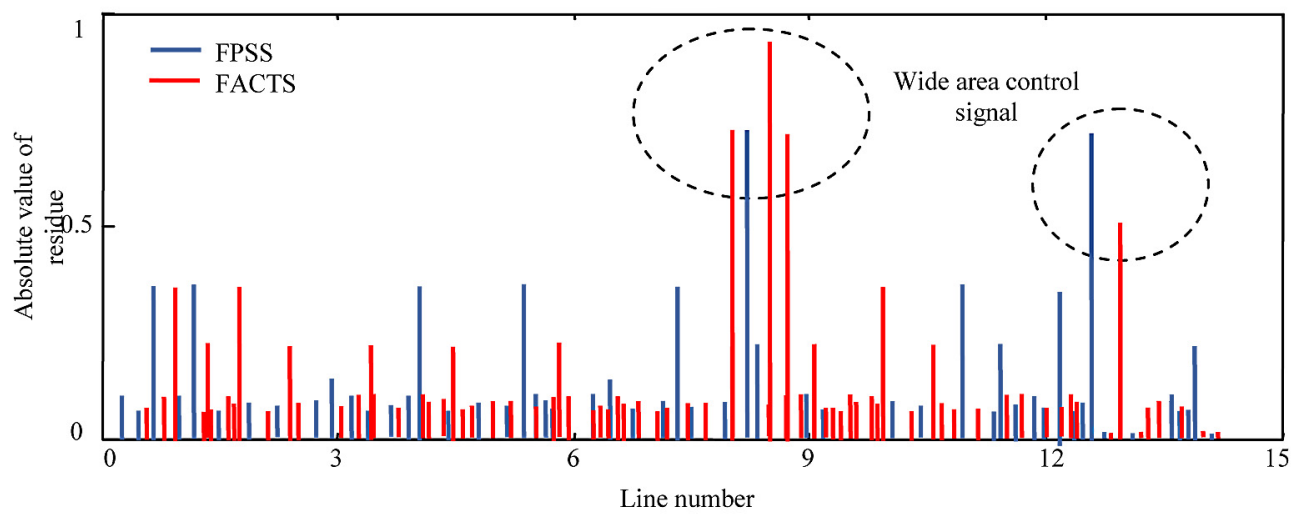

Fig. 9. Damping contribution of FPSS and FACTS devices using the available line current as the control input for inter-area oscillation modes. (a) Scenario 1. (b) Scenario 2.

area controller to damp out the inter-area modes of oscillations.

In this test the critical mode is categorized in to two mode as; Mode one: $-0.0046 \pm 3.4279 \mathrm{i}$ eigen-value, $0.25 \%$ damping by $0.5456(\mathrm{~Hz})$ frequency, Mode one: $-0.5297 \pm 6.8076 \mathrm{i}$ eigen-value, $7.76 \%$ damping by $1.0835(\mathrm{~Hz})$ frequency [8]. Where, there is only one inter-area mode of oscillation, of $0.5456(\mathrm{~Hz})$ in the system. This factor and categories is presented in Table 1 and Table 2 for second and third case studies, respectively.

Also, damping contribution of FPSS and FACTS devices using the available line current as the control input for inter-area oscillation modes has been presented in Figure 9. In this figure, (a) presents the changing the scenario 1 and (b) presents the changing in scenario 2 . These scenarios have been presented in the following for TAFM power system.

Also, in meta-heuristics algorithms the mature convergence can be occurred. To tackle the above-mentioned problem, this algorithm has been run 10 times over the proposed problem. Consequently, the best value of the consequence parameters of 10 runs are presented in Figure 10.

To demonstrate the effectiveness of the proposed strategy, this method has been tested over three case studies through the comparison of without wide area controller and [8]. The simulation results have been tested in different load con- 


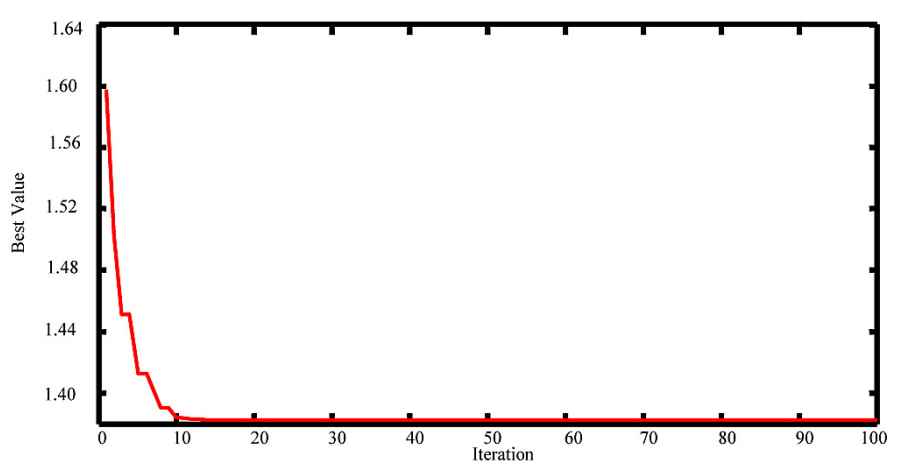

Fig. 10. Convergence trend of proposed algorithm

dition and faults where two scenarios have been considered to find the capability of the proposed wide area control strategy. The achieved results demonstrate the validity of the proposed method in comparison of other methods. For this purpose, at first we describe different scenarios in literature in the following way:

Scenario one. For the first scenario, the performance of the proposed controller under transient conditions is verified by applying a 3-cycle three-phase fault at $t=1 \mathrm{sec}$. This scenario is applied to bus 8 for Kundur's power system. The fault is cleared by permanent tripping the faulted line. The proposed strategy for wide-area control, has been compared with other model of widearea control [8] and without wide area controller. Figure 11 shows the speed deviations of G2 and G3 under nominal load condition. The obtained results demonstrate the validity of the proposed strategy where the overshoot $(O S)$, undershoot (US) and settling time (ST) of the proposed strategy has a better behavior in comparison of other models. The mentioned control criteria evaluated by the effectiveness of the proposed control strategy over generators deviations. Where, the mathematical equation of this criterion is presented in the following, which is named figure of demerit $(F D)$. Also, another criterion has been used for more consideration of proposed model capabilities as integral of time multiplied absolute value of the error (ITAE). This criterion presents q measure of deviations of generators and power system in response to the disturbance. A lower value of ITAE means lower deviations or a better system response indicating more effective performance of the control strategy. Load information of the presented power system is presented in [29].

Scenario two. For the second scenario, it is very important to test the proposed strategy under the loading power factor operating condition. For this purpose we simulate the load decreasing with a 0.2 p.u. step in mechanical torque at bus- 7 for $t=1.0$. The simulation results are presented in Figure 12 which demonstrates good performance of proposed control strategy. By considering this figure it can be said that the CPSS cannot provide
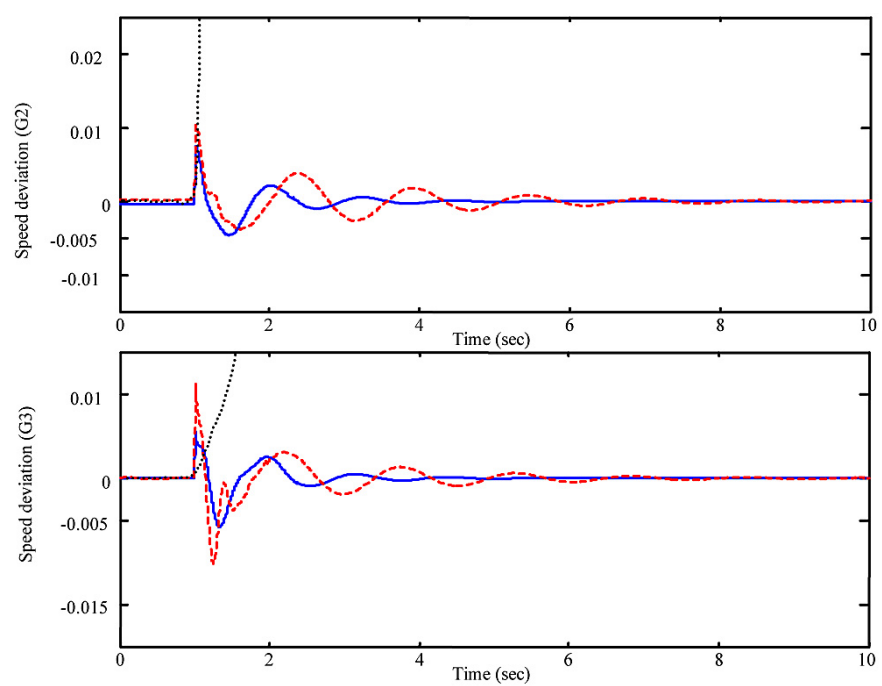

Fig. 11. System response under nominal load condition in scenario one of first case study; solid (proposed), dashed (WATSFDC), dotted (CPSS) 


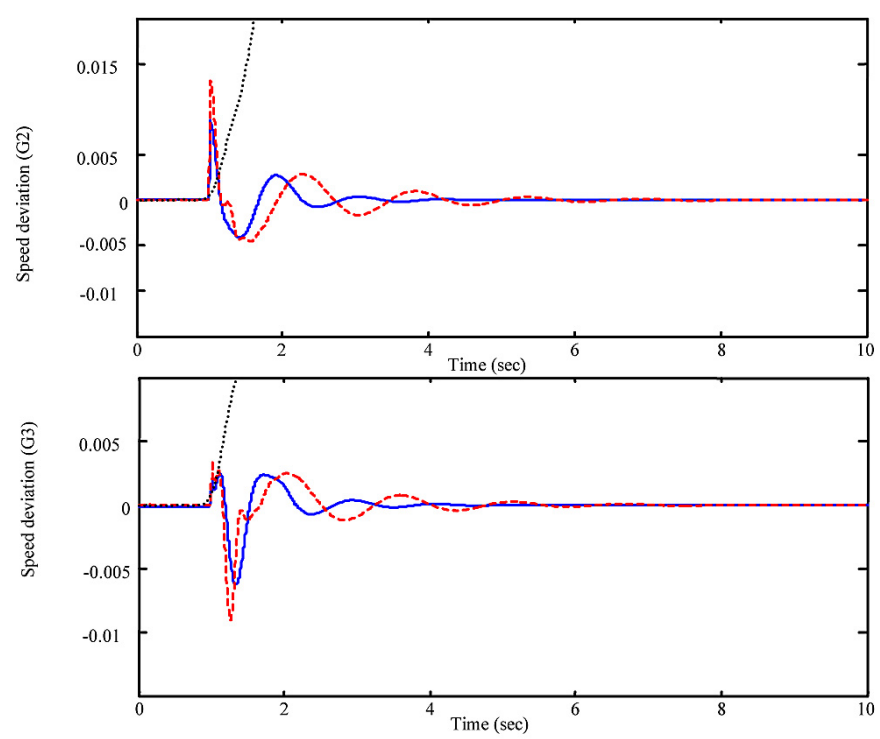

Fig. 12. System response under light load condition in scenario two of first case study; solid (proposed), dashed (WATSFDC), dotted (CPSS)

the stability of the mentioned power system. But, the other two strategies have good ST, OS and US. By scrutinizing these two methods, it is clear that the proposed strategy is better than WATSFDC [8].

$$
I T A E=\int_{0}^{t_{\text {sim }}}\left|\sum_{i=1}^{N_{g}} \omega_{i}\right| \cdot d t
$$

$F D=\sum_{i=1}^{N_{g}}\left(\left(\left|\max \left(\omega_{i}\right) \times 5000\right|\right)^{2}+\left(\left|\min \left(\omega_{i}\right) \times 10000\right|\right)^{2}+0.1 \times T_{s G i}^{2}\right)$

For the last part, tables of numerical results are presented through three load conditions of heavy, light and nominal (Tables 4, 5). By considering this information the superiority of the proposed strategy is obvious. Actually, we can change the optimization technique or the proposed controllers can be improved which leads to have a better control strategy. We would like to leave this part as a future work with more description.

Table 4. Value of ITAE in different scenarios

\begin{tabular}{|c|c|c|c|c|c|c|c|}
\hline \multirow{2}{*}{ Case } & \multirow{2}{*}{ Method } & \multicolumn{3}{|c|}{ Scenario One } & \multicolumn{3}{c|}{ Scenario Two } \\
\cline { 3 - 8 } & & $\mathrm{H}$ & $\mathrm{N}$ & $\mathrm{L}$ & $\mathrm{H}$ & $\mathrm{N}$ & $\mathrm{L}$ \\
\hline \multirow{2}{*}{ TAFM } & Proposed & 0.27 & 0.25 & 0.32 & 0.38 & 0.38 & 0.37 \\
\cline { 2 - 8 } & WATSFDC & 0.38 & 0.33 & 0.37 & 0.46 & 0.42 & 0.43 \\
\hline
\end{tabular}

$\mathrm{H}$ - heavy, $\mathrm{N}$ - nominal, L - light.

Table 5. Value of FD in different scenarios

\begin{tabular}{|c|c|c|c|c|c|c|c|}
\hline \multirow{2}{*}{ Case } & \multirow{2}{*}{ Method } & \multicolumn{3}{|c|}{ Scenario One } & \multicolumn{3}{c|}{ Scenario Two } \\
\cline { 3 - 8 } & & $\mathrm{H}$ & $\mathrm{N}$ & $\mathrm{L}$ & $\mathrm{H}$ & $\mathrm{N}$ & $\mathrm{L}$ \\
\hline \multirow{2}{*}{ TAFM } & Proposed & 0.98 & 0.90 & 0.92 & 0.99 & 0.97 & 0.98 \\
\cline { 2 - 8 } & WATSFDC & 1.03 & 0.98 & 1.01 & 1.01 & 0.98 & 1.03 \\
\hline
\end{tabular}

$\mathrm{H}$ - heavy, $\mathrm{N}$ - nominal, L - light.

\section{CONCLUSIONS}

In this paper coordination of FPSS and FACTS have been considered in a multi-machine power system. This strategy has been categorized in two main stages. In the first stage, the proposed new fuzzy control is presented to detect the widearea control signal. And in the second stage an intelligent algorithm is applied over fuzzy controller to partition the fuzzy space of the given input-output data. By the mentioned strategy, the application and robustness of fuzzy controller has been increased. Also, the global signal of the centralized controller is employed in wide area control scheme to damp out the inter-area mode as well as local mode of oscillations. Also, the parameters of FACTS controller have been evaluated by improved honey bee mating optimization (IHBMO). Effectiveness of the proposed method has been applied over two case studies of singlemachine infinite-bus (SMIB) and two areas four machine (TAFM) Kundur's power system. The obtained results demonstrate the superiority of the proposed strategy.

\section{REFERENCES}

1. Kamwa I., R. Grondin and Y. Hebert, Wide-area measurement based stabilizing control of large power systems - a decentralized/hierarchical approach. IEEE Trans. Power Syst., 16(1), 2001, 136-153.

2. Dotta D., A.S.E. Silva and I.C. Decker, Wide-area measurementbased two-level control design considering signals transmission delay," IEEE Trans. 
Power Syst., vol. 24, no. 1, pp. 208-216, Feb. 2009.

3. Majumder R., B.C. Pal, C. Dufour and P. Korba, Design and realtime implementation of robust FACTS controller for damping interarea oscillation. IEEE Trans. Power Syst., 21(2), 2006. 809-816.

4. Chaudhuri N.R., S. Ray, R. Majumder and B. Chaudhuri. A new approach to continuous latency compensation with adaptive phasor power oscillation damping controller (POD). IEEE Trans. Power Syst., 25(2), 2010, 939-946.

5. Farsangi M.M., Y.H. Song and K.Y. Lee, Choice of FACTS device control inputs for damping interarea oscillations. IEEE Trans. Power Syst., 19(2), 2004, 1135-1143.

6. Farsangi M.M., H. Nezamabadipour, Y.H. Song and K.Y. Lee, Placement of SVCs and selection of stabilizing signals in power systems. IEEE Trans. Power Syst., 22(3), 2007, 1061-1071.

7. Mao X.M., Y. Zhang, L. Guan and X.C.Wu, Coordinated control of inter-area oscillation in the China Southern power grid. IEEE Trans. Power Syst., 21(2), 2006. 845-852.

8. Padhy B.P., S.C. Srivastava and Nishchal K. Verma, Robust Wide-Area TS Fuzzy Output Feedback Controller for Enhancement of Stability in Multimachine Power System, IEEE Systems Journal, 6(3), 2012, 426-435.

9. Tanaka K. and H.O. Wang, Fuzzy control systems design and analysis: A linear matrix inequality approach. Wiley, New York 2001.

10. Feng G., A survey on analysis and design of model-based fuzzy control systems. IEEE Trans. Fuzzy Syst., 14(5), 2006, 676-697.

11. Delmotte F., T.M. Guerra, and M. Ksantini, Continuous Takagi-Sugeno's models: Reduction of the number of LMI conditions in various fuzzy control design technics. IEEE Trans. Fuzzy Syst., 15(3), 2007, 426-438.

12. Teixeira M.C.M. and S.H. Zak, Stabilizing controller design for uncertain nonlinear systems using fuzzy models. IEEE Trans. Fuzzy Syst., 7(2), 1999, 133-142.

13. Singh B., N.K. Sharma, A.N. Tiwari, K.S. Verma and S.N. Singh, Applications of phasor measurement units (PMUs) in electric power system networks incorporated with FACTS controllers. International Journal of Engineering, Science and Technology, 3(3), 2011, 64-82.

14. Chompoobutrgool Y., L. Vanfretti and M. Ghandhari, Survey on power system stabilizers control and their prospective applications for power system damping using synchrophasor-based widearea systems. European Transactions on Electrical Power, 21(8), 2011, 2098-2111.

15. IEEE Standard for Synchrophasors for Power Systems, IEEE, 2005, C37.118.

16. Yoneyama J., M. Nishikawa, H. Katayama and A.
Ichikawa, Design of output feedback controllers for Takagi-Sugeno fuzzy systems. Fuzzy Sets Systems, 121(1), 2001, 127-148.

17. Feng G., A survey on analysis and design of model-based fuzzy control systems. IEEE Trans. Fuzzy Syst., 14(5), 2006, 676-697.

18. Zhang Y. and A. Bose, Design of wide-area damping controllers for interarea oscillations. IEEE Trans. Power Syst., 23(3), 1136-1144.

19. Dong J., Y. Wang and G.-Hong Yang, Control Synthesis of Continuous-Time T-S Fuzzy Systems With Local Nonlinear Models. IEEE Transactions on Systems, Man, and Cybernetics - Part b: Cybernetics, 39(5), 2009, 1245-1258.

20. Zhao Q. and J. Jiang, Robust SVC controller design for improving power system damping. IEEE Trans. Power Syst., 10(4), 1995, 1927-1932.

21. Ray S. and G.K. Venayagamoorthy, Wide-area signal-based optimalneurocontroller for a UPFC. IEEE Trans. Power Del., 23(3), 2008, 1597-1605.

22. Abedinia O., M. Hajinasiri, M. Bekravi, A new stochastic search algorithm bundled honey bee mating for solving optimization problems. Neural Computing and Application, 25(7-8), 2014, 1921-1939.

23. Abedinia O., K. Kiani, A. Akbari, N. Amjady, H.A. Shayanfar, Improve honey bee mating optimization based on optimal congestion management in an electricity market. 8th International Conference on "Technical and Physical Problems of Power Engineering”, Norway 2012, 108-113.

24. Abedinia O., Mohammad. S. Naderi, A. Ghasemi, Robust LFC in Deregulated Environment: Fuzzy PID using HBMO. Proc. of the IEEE International Power \& Energy Society Power Systems Conference and Exposition, Italy, Rome 2011, 74-77.

25. Bekravi M., O. Abedinia, A new multi-objective meta heuristic algorithm based on environmental/ economic load dispatch with wind effect. Technical and Physical Problems of Engineering, 5(2), 15, 2013, 81-89.

26. Dai X.-D., D. He, L.-L. Fan, N.-H. Li and H. Chen, Improved ANN alpha; th-order inverse TCSC controller for enhancing power system transient stability. Proc. IET Gen. Transm. Distrib., 146(6), 1999, 550-556.

27. Abedinia O., M.S. Naderi, A. Jalili, B. Khamenepour, Optimal Tuning of multi-machine power system stabilizer parameters using genetic-algorithm. Proceedings of International Conference on Power System Technology, 24-28 October, 2010, 1-6.

28. Kundur P., Power system stability and control. 2nd ed. McGraw-Hill, New York, USA 2008.

29. Abedinia O., N. Amjady, H.R. Izadfar, H.A. Shayanfar, Multi-machine power system oscillation damping: Placement and tuning PSS VIA multi-objective HBMO. International Journal of Technical and Physical Problems of Engineering, 4(3), 12, 2012, 1-8. 